\title{
Non-Fregean Propositional Logic with Quantifiers
}

\author{
Joanna Golińska-Pilarek and Taneli Huuskonen
}

\begin{abstract}
We study the non-Fregean propositional logic with propositional quantifiers, denoted by $\mathrm{SCl}_{Q}$. We prove that $\mathrm{SCl}_{\mathrm{Q}}$ does not have the finite model property and that it is undecidable. We also present examples of how to interpret in $\mathrm{SCl}_{\mathrm{Q}}$ various mathematical theories, such as the theory of groups, rings, and fields, and we characterize the spectra of $\mathrm{SCl}_{\mathrm{Q}}$-sentences. Finally, we present a translation of $\mathrm{SCl}_{\mathrm{Q}}$ into a classical two-sorted first-order logic, and we use the translation to prove some model-theoretic properties of $\mathrm{SCl}_{\mathrm{Q}}$.
\end{abstract}

\section{Introduction}

Non-Fregean logics, introduced by Roman Suszko, can be seen as a realization of Gottlob Frege's semantic program with the exception of a postulate-known in the literature as the Fregean axiom - that treats the truth value of a sentence as its denotation. According to Frege, sentences are not only true or false, but are also names denoting the corresponding truth values. The Fregean axiom is a fundamental assumption underlying classical logic. The theory of models based on classical logic does not involve a universe corresponding to the statements in the appropriate language, but only assigns a simple truth value to each statement. Thus, the Fregean axiom is a formal expression of a certain philosophical view concerning the meanings of statements.

Non-Fregean logic was explicitly proposed by Suszko as an alternative to the established standard. Non-Fregean logic rejects the Fregean axiom and introduces a universe of the semantic correlates of statements, known as the universe of situations. In order to express claims concerning the universe of situations, a new connective $\equiv$, called the identity connective, is added to the language. The identity connective, as the name implies, expresses the identity of two statements; that is, it

Received January 21, 2013; accepted November 7, 2013

First published online February 9, 2016

2010 Mathematics Subject Classification: Primary 03B60; Secondary 03C80, 68Q19

Keywords: non-Fregean logic, sentential calculus with identity, identity connective, situational semantics

(C) 2016 by University of Notre Dame 10.1215/00294527-3470547 


\title{
Non-Fregean Propositional Logic with Quantifiers
}

\author{
Joanna Golińska-Pilarek and Taneli Huuskonen
}

\begin{abstract}
We study the non-Fregean propositional logic with propositional quantifiers, denoted by $\mathrm{SCl}_{Q}$. We prove that $\mathrm{SCl}_{\mathrm{Q}}$ does not have the finite model property and that it is undecidable. We also present examples of how to interpret in $\mathrm{SCl}_{\mathrm{Q}}$ various mathematical theories, such as the theory of groups, rings, and fields, and we characterize the spectra of $\mathrm{SCl}_{\mathrm{Q}}$-sentences. Finally, we present a translation of $\mathrm{SCl}_{\mathrm{Q}}$ into a classical two-sorted first-order logic, and we use the translation to prove some model-theoretic properties of $\mathrm{SCl}_{\mathrm{Q}}$.
\end{abstract}

\section{Introduction}

Non-Fregean logics, introduced by Roman Suszko, can be seen as a realization of Gottlob Frege's semantic program with the exception of a postulate-known in the literature as the Fregean axiom - that treats the truth value of a sentence as its denotation. According to Frege, sentences are not only true or false, but are also names denoting the corresponding truth values. The Fregean axiom is a fundamental assumption underlying classical logic. The theory of models based on classical logic does not involve a universe corresponding to the statements in the appropriate language, but only assigns a simple truth value to each statement. Thus, the Fregean axiom is a formal expression of a certain philosophical view concerning the meanings of statements.

Non-Fregean logic was explicitly proposed by Suszko as an alternative to the established standard. Non-Fregean logic rejects the Fregean axiom and introduces a universe of the semantic correlates of statements, known as the universe of situations. In order to express claims concerning the universe of situations, a new connective $\equiv$, called the identity connective, is added to the language. The identity connective, as the name implies, expresses the identity of two statements; that is, it

Received January 21, 2013; accepted November 7, 2013

First published online February 9, 2016

2010 Mathematics Subject Classification: Primary 03B60; Secondary 03C80, 68Q19

Keywords: non-Fregean logic, sentential calculus with identity, identity connective, situational semantics

(C) 2016 by University of Notre Dame 10.1215/00294527-3470547 


\title{
Non-Fregean Propositional Logic with Quantifiers
}

\author{
Joanna Golińska-Pilarek and Taneli Huuskonen
}

\begin{abstract}
We study the non-Fregean propositional logic with propositional quantifiers, denoted by $\mathrm{SCl}_{Q}$. We prove that $\mathrm{SCl}_{\mathrm{Q}}$ does not have the finite model property and that it is undecidable. We also present examples of how to interpret in $\mathrm{SCl}_{\mathrm{Q}}$ various mathematical theories, such as the theory of groups, rings, and fields, and we characterize the spectra of $\mathrm{SCl}_{\mathrm{Q}}$-sentences. Finally, we present a translation of $\mathrm{SCl}_{\mathrm{Q}}$ into a classical two-sorted first-order logic, and we use the translation to prove some model-theoretic properties of $\mathrm{SCl}_{\mathrm{Q}}$.
\end{abstract}

\section{Introduction}

Non-Fregean logics, introduced by Roman Suszko, can be seen as a realization of Gottlob Frege's semantic program with the exception of a postulate-known in the literature as the Fregean axiom - that treats the truth value of a sentence as its denotation. According to Frege, sentences are not only true or false, but are also names denoting the corresponding truth values. The Fregean axiom is a fundamental assumption underlying classical logic. The theory of models based on classical logic does not involve a universe corresponding to the statements in the appropriate language, but only assigns a simple truth value to each statement. Thus, the Fregean axiom is a formal expression of a certain philosophical view concerning the meanings of statements.

Non-Fregean logic was explicitly proposed by Suszko as an alternative to the established standard. Non-Fregean logic rejects the Fregean axiom and introduces a universe of the semantic correlates of statements, known as the universe of situations. In order to express claims concerning the universe of situations, a new connective $\equiv$, called the identity connective, is added to the language. The identity connective, as the name implies, expresses the identity of two statements; that is, it

Received January 21, 2013; accepted November 7, 2013

First published online February 9, 2016

2010 Mathematics Subject Classification: Primary 03B60; Secondary 03C80, 68Q19

Keywords: non-Fregean logic, sentential calculus with identity, identity connective, situational semantics

(C) 2016 by University of Notre Dame 10.1215/00294527-3470547 


\title{
Non-Fregean Propositional Logic with Quantifiers
}

\author{
Joanna Golińska-Pilarek and Taneli Huuskonen
}

\begin{abstract}
We study the non-Fregean propositional logic with propositional quantifiers, denoted by $\mathrm{SCl}_{Q}$. We prove that $\mathrm{SCl}_{\mathrm{Q}}$ does not have the finite model property and that it is undecidable. We also present examples of how to interpret in $\mathrm{SCl}_{\mathrm{Q}}$ various mathematical theories, such as the theory of groups, rings, and fields, and we characterize the spectra of $\mathrm{SCl}_{\mathrm{Q}}$-sentences. Finally, we present a translation of $\mathrm{SCl}_{\mathrm{Q}}$ into a classical two-sorted first-order logic, and we use the translation to prove some model-theoretic properties of $\mathrm{SCl}_{\mathrm{Q}}$.
\end{abstract}

\section{Introduction}

Non-Fregean logics, introduced by Roman Suszko, can be seen as a realization of Gottlob Frege's semantic program with the exception of a postulate-known in the literature as the Fregean axiom - that treats the truth value of a sentence as its denotation. According to Frege, sentences are not only true or false, but are also names denoting the corresponding truth values. The Fregean axiom is a fundamental assumption underlying classical logic. The theory of models based on classical logic does not involve a universe corresponding to the statements in the appropriate language, but only assigns a simple truth value to each statement. Thus, the Fregean axiom is a formal expression of a certain philosophical view concerning the meanings of statements.

Non-Fregean logic was explicitly proposed by Suszko as an alternative to the established standard. Non-Fregean logic rejects the Fregean axiom and introduces a universe of the semantic correlates of statements, known as the universe of situations. In order to express claims concerning the universe of situations, a new connective $\equiv$, called the identity connective, is added to the language. The identity connective, as the name implies, expresses the identity of two statements; that is, it

Received January 21, 2013; accepted November 7, 2013

First published online February 9, 2016

2010 Mathematics Subject Classification: Primary 03B60; Secondary 03C80, 68Q19

Keywords: non-Fregean logic, sentential calculus with identity, identity connective, situational semantics

(C) 2016 by University of Notre Dame 10.1215/00294527-3470547 


\title{
Non-Fregean Propositional Logic with Quantifiers
}

\author{
Joanna Golińska-Pilarek and Taneli Huuskonen
}

\begin{abstract}
We study the non-Fregean propositional logic with propositional quantifiers, denoted by $\mathrm{SCl}_{Q}$. We prove that $\mathrm{SCl}_{\mathrm{Q}}$ does not have the finite model property and that it is undecidable. We also present examples of how to interpret in $\mathrm{SCl}_{\mathrm{Q}}$ various mathematical theories, such as the theory of groups, rings, and fields, and we characterize the spectra of $\mathrm{SCl}_{\mathrm{Q}}$-sentences. Finally, we present a translation of $\mathrm{SCl}_{\mathrm{Q}}$ into a classical two-sorted first-order logic, and we use the translation to prove some model-theoretic properties of $\mathrm{SCl}_{\mathrm{Q}}$.
\end{abstract}

\section{Introduction}

Non-Fregean logics, introduced by Roman Suszko, can be seen as a realization of Gottlob Frege's semantic program with the exception of a postulate-known in the literature as the Fregean axiom - that treats the truth value of a sentence as its denotation. According to Frege, sentences are not only true or false, but are also names denoting the corresponding truth values. The Fregean axiom is a fundamental assumption underlying classical logic. The theory of models based on classical logic does not involve a universe corresponding to the statements in the appropriate language, but only assigns a simple truth value to each statement. Thus, the Fregean axiom is a formal expression of a certain philosophical view concerning the meanings of statements.

Non-Fregean logic was explicitly proposed by Suszko as an alternative to the established standard. Non-Fregean logic rejects the Fregean axiom and introduces a universe of the semantic correlates of statements, known as the universe of situations. In order to express claims concerning the universe of situations, a new connective $\equiv$, called the identity connective, is added to the language. The identity connective, as the name implies, expresses the identity of two statements; that is, it

Received January 21, 2013; accepted November 7, 2013

First published online February 9, 2016

2010 Mathematics Subject Classification: Primary 03B60; Secondary 03C80, 68Q19

Keywords: non-Fregean logic, sentential calculus with identity, identity connective, situational semantics

(C) 2016 by University of Notre Dame 10.1215/00294527-3470547 


\title{
Non-Fregean Propositional Logic with Quantifiers
}

\author{
Joanna Golińska-Pilarek and Taneli Huuskonen
}

\begin{abstract}
We study the non-Fregean propositional logic with propositional quantifiers, denoted by $\mathrm{SCl}_{Q}$. We prove that $\mathrm{SCl}_{\mathrm{Q}}$ does not have the finite model property and that it is undecidable. We also present examples of how to interpret in $\mathrm{SCl}_{\mathrm{Q}}$ various mathematical theories, such as the theory of groups, rings, and fields, and we characterize the spectra of $\mathrm{SCl}_{\mathrm{Q}}$-sentences. Finally, we present a translation of $\mathrm{SCl}_{\mathrm{Q}}$ into a classical two-sorted first-order logic, and we use the translation to prove some model-theoretic properties of $\mathrm{SCl}_{\mathrm{Q}}$.
\end{abstract}

\section{Introduction}

Non-Fregean logics, introduced by Roman Suszko, can be seen as a realization of Gottlob Frege's semantic program with the exception of a postulate-known in the literature as the Fregean axiom - that treats the truth value of a sentence as its denotation. According to Frege, sentences are not only true or false, but are also names denoting the corresponding truth values. The Fregean axiom is a fundamental assumption underlying classical logic. The theory of models based on classical logic does not involve a universe corresponding to the statements in the appropriate language, but only assigns a simple truth value to each statement. Thus, the Fregean axiom is a formal expression of a certain philosophical view concerning the meanings of statements.

Non-Fregean logic was explicitly proposed by Suszko as an alternative to the established standard. Non-Fregean logic rejects the Fregean axiom and introduces a universe of the semantic correlates of statements, known as the universe of situations. In order to express claims concerning the universe of situations, a new connective $\equiv$, called the identity connective, is added to the language. The identity connective, as the name implies, expresses the identity of two statements; that is, it

Received January 21, 2013; accepted November 7, 2013

First published online February 9, 2016

2010 Mathematics Subject Classification: Primary 03B60; Secondary 03C80, 68Q19

Keywords: non-Fregean logic, sentential calculus with identity, identity connective, situational semantics

(C) 2016 by University of Notre Dame 10.1215/00294527-3470547 


\title{
Non-Fregean Propositional Logic with Quantifiers
}

\author{
Joanna Golińska-Pilarek and Taneli Huuskonen
}

\begin{abstract}
We study the non-Fregean propositional logic with propositional quantifiers, denoted by $\mathrm{SCl}_{Q}$. We prove that $\mathrm{SCl}_{\mathrm{Q}}$ does not have the finite model property and that it is undecidable. We also present examples of how to interpret in $\mathrm{SCl}_{\mathrm{Q}}$ various mathematical theories, such as the theory of groups, rings, and fields, and we characterize the spectra of $\mathrm{SCl}_{\mathrm{Q}}$-sentences. Finally, we present a translation of $\mathrm{SCl}_{\mathrm{Q}}$ into a classical two-sorted first-order logic, and we use the translation to prove some model-theoretic properties of $\mathrm{SCl}_{\mathrm{Q}}$.
\end{abstract}

\section{Introduction}

Non-Fregean logics, introduced by Roman Suszko, can be seen as a realization of Gottlob Frege's semantic program with the exception of a postulate-known in the literature as the Fregean axiom - that treats the truth value of a sentence as its denotation. According to Frege, sentences are not only true or false, but are also names denoting the corresponding truth values. The Fregean axiom is a fundamental assumption underlying classical logic. The theory of models based on classical logic does not involve a universe corresponding to the statements in the appropriate language, but only assigns a simple truth value to each statement. Thus, the Fregean axiom is a formal expression of a certain philosophical view concerning the meanings of statements.

Non-Fregean logic was explicitly proposed by Suszko as an alternative to the established standard. Non-Fregean logic rejects the Fregean axiom and introduces a universe of the semantic correlates of statements, known as the universe of situations. In order to express claims concerning the universe of situations, a new connective $\equiv$, called the identity connective, is added to the language. The identity connective, as the name implies, expresses the identity of two statements; that is, it

Received January 21, 2013; accepted November 7, 2013

First published online February 9, 2016

2010 Mathematics Subject Classification: Primary 03B60; Secondary 03C80, 68Q19

Keywords: non-Fregean logic, sentential calculus with identity, identity connective, situational semantics

(C) 2016 by University of Notre Dame 10.1215/00294527-3470547 


\title{
Non-Fregean Propositional Logic with Quantifiers
}

\author{
Joanna Golińska-Pilarek and Taneli Huuskonen
}

\begin{abstract}
We study the non-Fregean propositional logic with propositional quantifiers, denoted by $\mathrm{SCl}_{Q}$. We prove that $\mathrm{SCl}_{\mathrm{Q}}$ does not have the finite model property and that it is undecidable. We also present examples of how to interpret in $\mathrm{SCl}_{\mathrm{Q}}$ various mathematical theories, such as the theory of groups, rings, and fields, and we characterize the spectra of $\mathrm{SCl}_{\mathrm{Q}}$-sentences. Finally, we present a translation of $\mathrm{SCl}_{\mathrm{Q}}$ into a classical two-sorted first-order logic, and we use the translation to prove some model-theoretic properties of $\mathrm{SCl}_{\mathrm{Q}}$.
\end{abstract}

\section{Introduction}

Non-Fregean logics, introduced by Roman Suszko, can be seen as a realization of Gottlob Frege's semantic program with the exception of a postulate-known in the literature as the Fregean axiom - that treats the truth value of a sentence as its denotation. According to Frege, sentences are not only true or false, but are also names denoting the corresponding truth values. The Fregean axiom is a fundamental assumption underlying classical logic. The theory of models based on classical logic does not involve a universe corresponding to the statements in the appropriate language, but only assigns a simple truth value to each statement. Thus, the Fregean axiom is a formal expression of a certain philosophical view concerning the meanings of statements.

Non-Fregean logic was explicitly proposed by Suszko as an alternative to the established standard. Non-Fregean logic rejects the Fregean axiom and introduces a universe of the semantic correlates of statements, known as the universe of situations. In order to express claims concerning the universe of situations, a new connective $\equiv$, called the identity connective, is added to the language. The identity connective, as the name implies, expresses the identity of two statements; that is, it

Received January 21, 2013; accepted November 7, 2013

First published online February 9, 2016

2010 Mathematics Subject Classification: Primary 03B60; Secondary 03C80, 68Q19

Keywords: non-Fregean logic, sentential calculus with identity, identity connective, situational semantics

(C) 2016 by University of Notre Dame 10.1215/00294527-3470547 


\title{
Non-Fregean Propositional Logic with Quantifiers
}

\author{
Joanna Golińska-Pilarek and Taneli Huuskonen
}

\begin{abstract}
We study the non-Fregean propositional logic with propositional quantifiers, denoted by $\mathrm{SCl}_{Q}$. We prove that $\mathrm{SCl}_{\mathrm{Q}}$ does not have the finite model property and that it is undecidable. We also present examples of how to interpret in $\mathrm{SCl}_{\mathrm{Q}}$ various mathematical theories, such as the theory of groups, rings, and fields, and we characterize the spectra of $\mathrm{SCl}_{\mathrm{Q}}$-sentences. Finally, we present a translation of $\mathrm{SCl}_{\mathrm{Q}}$ into a classical two-sorted first-order logic, and we use the translation to prove some model-theoretic properties of $\mathrm{SCl}_{\mathrm{Q}}$.
\end{abstract}

\section{Introduction}

Non-Fregean logics, introduced by Roman Suszko, can be seen as a realization of Gottlob Frege's semantic program with the exception of a postulate-known in the literature as the Fregean axiom - that treats the truth value of a sentence as its denotation. According to Frege, sentences are not only true or false, but are also names denoting the corresponding truth values. The Fregean axiom is a fundamental assumption underlying classical logic. The theory of models based on classical logic does not involve a universe corresponding to the statements in the appropriate language, but only assigns a simple truth value to each statement. Thus, the Fregean axiom is a formal expression of a certain philosophical view concerning the meanings of statements.

Non-Fregean logic was explicitly proposed by Suszko as an alternative to the established standard. Non-Fregean logic rejects the Fregean axiom and introduces a universe of the semantic correlates of statements, known as the universe of situations. In order to express claims concerning the universe of situations, a new connective $\equiv$, called the identity connective, is added to the language. The identity connective, as the name implies, expresses the identity of two statements; that is, it

Received January 21, 2013; accepted November 7, 2013

First published online February 9, 2016

2010 Mathematics Subject Classification: Primary 03B60; Secondary 03C80, 68Q19

Keywords: non-Fregean logic, sentential calculus with identity, identity connective, situational semantics

(C) 2016 by University of Notre Dame 10.1215/00294527-3470547 


\title{
Non-Fregean Propositional Logic with Quantifiers
}

\author{
Joanna Golińska-Pilarek and Taneli Huuskonen
}

\begin{abstract}
We study the non-Fregean propositional logic with propositional quantifiers, denoted by $\mathrm{SCl}_{Q}$. We prove that $\mathrm{SCl}_{\mathrm{Q}}$ does not have the finite model property and that it is undecidable. We also present examples of how to interpret in $\mathrm{SCl}_{\mathrm{Q}}$ various mathematical theories, such as the theory of groups, rings, and fields, and we characterize the spectra of $\mathrm{SCl}_{\mathrm{Q}}$-sentences. Finally, we present a translation of $\mathrm{SCl}_{\mathrm{Q}}$ into a classical two-sorted first-order logic, and we use the translation to prove some model-theoretic properties of $\mathrm{SCl}_{\mathrm{Q}}$.
\end{abstract}

\section{Introduction}

Non-Fregean logics, introduced by Roman Suszko, can be seen as a realization of Gottlob Frege's semantic program with the exception of a postulate-known in the literature as the Fregean axiom - that treats the truth value of a sentence as its denotation. According to Frege, sentences are not only true or false, but are also names denoting the corresponding truth values. The Fregean axiom is a fundamental assumption underlying classical logic. The theory of models based on classical logic does not involve a universe corresponding to the statements in the appropriate language, but only assigns a simple truth value to each statement. Thus, the Fregean axiom is a formal expression of a certain philosophical view concerning the meanings of statements.

Non-Fregean logic was explicitly proposed by Suszko as an alternative to the established standard. Non-Fregean logic rejects the Fregean axiom and introduces a universe of the semantic correlates of statements, known as the universe of situations. In order to express claims concerning the universe of situations, a new connective $\equiv$, called the identity connective, is added to the language. The identity connective, as the name implies, expresses the identity of two statements; that is, it

Received January 21, 2013; accepted November 7, 2013

First published online February 9, 2016

2010 Mathematics Subject Classification: Primary 03B60; Secondary 03C80, 68Q19

Keywords: non-Fregean logic, sentential calculus with identity, identity connective, situational semantics

(C) 2016 by University of Notre Dame 10.1215/00294527-3470547 


\title{
Non-Fregean Propositional Logic with Quantifiers
}

\author{
Joanna Golińska-Pilarek and Taneli Huuskonen
}

\begin{abstract}
We study the non-Fregean propositional logic with propositional quantifiers, denoted by $\mathrm{SCl}_{Q}$. We prove that $\mathrm{SCl}_{\mathrm{Q}}$ does not have the finite model property and that it is undecidable. We also present examples of how to interpret in $\mathrm{SCl}_{\mathrm{Q}}$ various mathematical theories, such as the theory of groups, rings, and fields, and we characterize the spectra of $\mathrm{SCl}_{\mathrm{Q}}$-sentences. Finally, we present a translation of $\mathrm{SCl}_{\mathrm{Q}}$ into a classical two-sorted first-order logic, and we use the translation to prove some model-theoretic properties of $\mathrm{SCl}_{\mathrm{Q}}$.
\end{abstract}

\section{Introduction}

Non-Fregean logics, introduced by Roman Suszko, can be seen as a realization of Gottlob Frege's semantic program with the exception of a postulate-known in the literature as the Fregean axiom - that treats the truth value of a sentence as its denotation. According to Frege, sentences are not only true or false, but are also names denoting the corresponding truth values. The Fregean axiom is a fundamental assumption underlying classical logic. The theory of models based on classical logic does not involve a universe corresponding to the statements in the appropriate language, but only assigns a simple truth value to each statement. Thus, the Fregean axiom is a formal expression of a certain philosophical view concerning the meanings of statements.

Non-Fregean logic was explicitly proposed by Suszko as an alternative to the established standard. Non-Fregean logic rejects the Fregean axiom and introduces a universe of the semantic correlates of statements, known as the universe of situations. In order to express claims concerning the universe of situations, a new connective $\equiv$, called the identity connective, is added to the language. The identity connective, as the name implies, expresses the identity of two statements; that is, it

Received January 21, 2013; accepted November 7, 2013

First published online February 9, 2016

2010 Mathematics Subject Classification: Primary 03B60; Secondary 03C80, 68Q19

Keywords: non-Fregean logic, sentential calculus with identity, identity connective, situational semantics

(C) 2016 by University of Notre Dame 10.1215/00294527-3470547 


\title{
Non-Fregean Propositional Logic with Quantifiers
}

\author{
Joanna Golińska-Pilarek and Taneli Huuskonen
}

\begin{abstract}
We study the non-Fregean propositional logic with propositional quantifiers, denoted by $\mathrm{SCl}_{Q}$. We prove that $\mathrm{SCl}_{\mathrm{Q}}$ does not have the finite model property and that it is undecidable. We also present examples of how to interpret in $\mathrm{SCl}_{\mathrm{Q}}$ various mathematical theories, such as the theory of groups, rings, and fields, and we characterize the spectra of $\mathrm{SCl}_{\mathrm{Q}}$-sentences. Finally, we present a translation of $\mathrm{SCl}_{\mathrm{Q}}$ into a classical two-sorted first-order logic, and we use the translation to prove some model-theoretic properties of $\mathrm{SCl}_{\mathrm{Q}}$.
\end{abstract}

\section{Introduction}

Non-Fregean logics, introduced by Roman Suszko, can be seen as a realization of Gottlob Frege's semantic program with the exception of a postulate-known in the literature as the Fregean axiom - that treats the truth value of a sentence as its denotation. According to Frege, sentences are not only true or false, but are also names denoting the corresponding truth values. The Fregean axiom is a fundamental assumption underlying classical logic. The theory of models based on classical logic does not involve a universe corresponding to the statements in the appropriate language, but only assigns a simple truth value to each statement. Thus, the Fregean axiom is a formal expression of a certain philosophical view concerning the meanings of statements.

Non-Fregean logic was explicitly proposed by Suszko as an alternative to the established standard. Non-Fregean logic rejects the Fregean axiom and introduces a universe of the semantic correlates of statements, known as the universe of situations. In order to express claims concerning the universe of situations, a new connective $\equiv$, called the identity connective, is added to the language. The identity connective, as the name implies, expresses the identity of two statements; that is, it

Received January 21, 2013; accepted November 7, 2013

First published online February 9, 2016

2010 Mathematics Subject Classification: Primary 03B60; Secondary 03C80, 68Q19

Keywords: non-Fregean logic, sentential calculus with identity, identity connective, situational semantics

(C) 2016 by University of Notre Dame 10.1215/00294527-3470547 


\title{
Non-Fregean Propositional Logic with Quantifiers
}

\author{
Joanna Golińska-Pilarek and Taneli Huuskonen
}

\begin{abstract}
We study the non-Fregean propositional logic with propositional quantifiers, denoted by $\mathrm{SCl}_{Q}$. We prove that $\mathrm{SCl}_{\mathrm{Q}}$ does not have the finite model property and that it is undecidable. We also present examples of how to interpret in $\mathrm{SCl}_{\mathrm{Q}}$ various mathematical theories, such as the theory of groups, rings, and fields, and we characterize the spectra of $\mathrm{SCl}_{\mathrm{Q}}$-sentences. Finally, we present a translation of $\mathrm{SCl}_{\mathrm{Q}}$ into a classical two-sorted first-order logic, and we use the translation to prove some model-theoretic properties of $\mathrm{SCl}_{\mathrm{Q}}$.
\end{abstract}

\section{Introduction}

Non-Fregean logics, introduced by Roman Suszko, can be seen as a realization of Gottlob Frege's semantic program with the exception of a postulate-known in the literature as the Fregean axiom - that treats the truth value of a sentence as its denotation. According to Frege, sentences are not only true or false, but are also names denoting the corresponding truth values. The Fregean axiom is a fundamental assumption underlying classical logic. The theory of models based on classical logic does not involve a universe corresponding to the statements in the appropriate language, but only assigns a simple truth value to each statement. Thus, the Fregean axiom is a formal expression of a certain philosophical view concerning the meanings of statements.

Non-Fregean logic was explicitly proposed by Suszko as an alternative to the established standard. Non-Fregean logic rejects the Fregean axiom and introduces a universe of the semantic correlates of statements, known as the universe of situations. In order to express claims concerning the universe of situations, a new connective $\equiv$, called the identity connective, is added to the language. The identity connective, as the name implies, expresses the identity of two statements; that is, it

Received January 21, 2013; accepted November 7, 2013

First published online February 9, 2016

2010 Mathematics Subject Classification: Primary 03B60; Secondary 03C80, 68Q19

Keywords: non-Fregean logic, sentential calculus with identity, identity connective, situational semantics

(C) 2016 by University of Notre Dame 10.1215/00294527-3470547 


\title{
Non-Fregean Propositional Logic with Quantifiers
}

\author{
Joanna Golińska-Pilarek and Taneli Huuskonen
}

\begin{abstract}
We study the non-Fregean propositional logic with propositional quantifiers, denoted by $\mathrm{SCl}_{Q}$. We prove that $\mathrm{SCl}_{\mathrm{Q}}$ does not have the finite model property and that it is undecidable. We also present examples of how to interpret in $\mathrm{SCl}_{\mathrm{Q}}$ various mathematical theories, such as the theory of groups, rings, and fields, and we characterize the spectra of $\mathrm{SCl}_{\mathrm{Q}}$-sentences. Finally, we present a translation of $\mathrm{SCl}_{\mathrm{Q}}$ into a classical two-sorted first-order logic, and we use the translation to prove some model-theoretic properties of $\mathrm{SCl}_{\mathrm{Q}}$.
\end{abstract}

\section{Introduction}

Non-Fregean logics, introduced by Roman Suszko, can be seen as a realization of Gottlob Frege's semantic program with the exception of a postulate-known in the literature as the Fregean axiom - that treats the truth value of a sentence as its denotation. According to Frege, sentences are not only true or false, but are also names denoting the corresponding truth values. The Fregean axiom is a fundamental assumption underlying classical logic. The theory of models based on classical logic does not involve a universe corresponding to the statements in the appropriate language, but only assigns a simple truth value to each statement. Thus, the Fregean axiom is a formal expression of a certain philosophical view concerning the meanings of statements.

Non-Fregean logic was explicitly proposed by Suszko as an alternative to the established standard. Non-Fregean logic rejects the Fregean axiom and introduces a universe of the semantic correlates of statements, known as the universe of situations. In order to express claims concerning the universe of situations, a new connective $\equiv$, called the identity connective, is added to the language. The identity connective, as the name implies, expresses the identity of two statements; that is, it

Received January 21, 2013; accepted November 7, 2013

First published online February 9, 2016

2010 Mathematics Subject Classification: Primary 03B60; Secondary 03C80, 68Q19

Keywords: non-Fregean logic, sentential calculus with identity, identity connective, situational semantics

(C) 2016 by University of Notre Dame 10.1215/00294527-3470547 


\title{
Non-Fregean Propositional Logic with Quantifiers
}

\author{
Joanna Golińska-Pilarek and Taneli Huuskonen
}

\begin{abstract}
We study the non-Fregean propositional logic with propositional quantifiers, denoted by $\mathrm{SCl}_{Q}$. We prove that $\mathrm{SCl}_{\mathrm{Q}}$ does not have the finite model property and that it is undecidable. We also present examples of how to interpret in $\mathrm{SCl}_{\mathrm{Q}}$ various mathematical theories, such as the theory of groups, rings, and fields, and we characterize the spectra of $\mathrm{SCl}_{\mathrm{Q}}$-sentences. Finally, we present a translation of $\mathrm{SCl}_{\mathrm{Q}}$ into a classical two-sorted first-order logic, and we use the translation to prove some model-theoretic properties of $\mathrm{SCl}_{\mathrm{Q}}$.
\end{abstract}

\section{Introduction}

Non-Fregean logics, introduced by Roman Suszko, can be seen as a realization of Gottlob Frege's semantic program with the exception of a postulate-known in the literature as the Fregean axiom - that treats the truth value of a sentence as its denotation. According to Frege, sentences are not only true or false, but are also names denoting the corresponding truth values. The Fregean axiom is a fundamental assumption underlying classical logic. The theory of models based on classical logic does not involve a universe corresponding to the statements in the appropriate language, but only assigns a simple truth value to each statement. Thus, the Fregean axiom is a formal expression of a certain philosophical view concerning the meanings of statements.

Non-Fregean logic was explicitly proposed by Suszko as an alternative to the established standard. Non-Fregean logic rejects the Fregean axiom and introduces a universe of the semantic correlates of statements, known as the universe of situations. In order to express claims concerning the universe of situations, a new connective $\equiv$, called the identity connective, is added to the language. The identity connective, as the name implies, expresses the identity of two statements; that is, it

Received January 21, 2013; accepted November 7, 2013

First published online February 9, 2016

2010 Mathematics Subject Classification: Primary 03B60; Secondary 03C80, 68Q19

Keywords: non-Fregean logic, sentential calculus with identity, identity connective, situational semantics

(C) 2016 by University of Notre Dame 10.1215/00294527-3470547 


\title{
Non-Fregean Propositional Logic with Quantifiers
}

\author{
Joanna Golińska-Pilarek and Taneli Huuskonen
}

\begin{abstract}
We study the non-Fregean propositional logic with propositional quantifiers, denoted by $\mathrm{SCl}_{Q}$. We prove that $\mathrm{SCl}_{\mathrm{Q}}$ does not have the finite model property and that it is undecidable. We also present examples of how to interpret in $\mathrm{SCl}_{\mathrm{Q}}$ various mathematical theories, such as the theory of groups, rings, and fields, and we characterize the spectra of $\mathrm{SCl}_{\mathrm{Q}}$-sentences. Finally, we present a translation of $\mathrm{SCl}_{\mathrm{Q}}$ into a classical two-sorted first-order logic, and we use the translation to prove some model-theoretic properties of $\mathrm{SCl}_{\mathrm{Q}}$.
\end{abstract}

\section{Introduction}

Non-Fregean logics, introduced by Roman Suszko, can be seen as a realization of Gottlob Frege's semantic program with the exception of a postulate-known in the literature as the Fregean axiom - that treats the truth value of a sentence as its denotation. According to Frege, sentences are not only true or false, but are also names denoting the corresponding truth values. The Fregean axiom is a fundamental assumption underlying classical logic. The theory of models based on classical logic does not involve a universe corresponding to the statements in the appropriate language, but only assigns a simple truth value to each statement. Thus, the Fregean axiom is a formal expression of a certain philosophical view concerning the meanings of statements.

Non-Fregean logic was explicitly proposed by Suszko as an alternative to the established standard. Non-Fregean logic rejects the Fregean axiom and introduces a universe of the semantic correlates of statements, known as the universe of situations. In order to express claims concerning the universe of situations, a new connective $\equiv$, called the identity connective, is added to the language. The identity connective, as the name implies, expresses the identity of two statements; that is, it

Received January 21, 2013; accepted November 7, 2013

First published online February 9, 2016

2010 Mathematics Subject Classification: Primary 03B60; Secondary 03C80, 68Q19

Keywords: non-Fregean logic, sentential calculus with identity, identity connective, situational semantics

(C) 2016 by University of Notre Dame 10.1215/00294527-3470547 


\title{
Non-Fregean Propositional Logic with Quantifiers
}

\author{
Joanna Golińska-Pilarek and Taneli Huuskonen
}

\begin{abstract}
We study the non-Fregean propositional logic with propositional quantifiers, denoted by $\mathrm{SCl}_{Q}$. We prove that $\mathrm{SCl}_{\mathrm{Q}}$ does not have the finite model property and that it is undecidable. We also present examples of how to interpret in $\mathrm{SCl}_{\mathrm{Q}}$ various mathematical theories, such as the theory of groups, rings, and fields, and we characterize the spectra of $\mathrm{SCl}_{\mathrm{Q}}$-sentences. Finally, we present a translation of $\mathrm{SCl}_{\mathrm{Q}}$ into a classical two-sorted first-order logic, and we use the translation to prove some model-theoretic properties of $\mathrm{SCl}_{\mathrm{Q}}$.
\end{abstract}

\section{Introduction}

Non-Fregean logics, introduced by Roman Suszko, can be seen as a realization of Gottlob Frege's semantic program with the exception of a postulate-known in the literature as the Fregean axiom - that treats the truth value of a sentence as its denotation. According to Frege, sentences are not only true or false, but are also names denoting the corresponding truth values. The Fregean axiom is a fundamental assumption underlying classical logic. The theory of models based on classical logic does not involve a universe corresponding to the statements in the appropriate language, but only assigns a simple truth value to each statement. Thus, the Fregean axiom is a formal expression of a certain philosophical view concerning the meanings of statements.

Non-Fregean logic was explicitly proposed by Suszko as an alternative to the established standard. Non-Fregean logic rejects the Fregean axiom and introduces a universe of the semantic correlates of statements, known as the universe of situations. In order to express claims concerning the universe of situations, a new connective $\equiv$, called the identity connective, is added to the language. The identity connective, as the name implies, expresses the identity of two statements; that is, it

Received January 21, 2013; accepted November 7, 2013

First published online February 9, 2016

2010 Mathematics Subject Classification: Primary 03B60; Secondary 03C80, 68Q19

Keywords: non-Fregean logic, sentential calculus with identity, identity connective, situational semantics

(C) 2016 by University of Notre Dame 10.1215/00294527-3470547 


\title{
Non-Fregean Propositional Logic with Quantifiers
}

\author{
Joanna Golińska-Pilarek and Taneli Huuskonen
}

\begin{abstract}
We study the non-Fregean propositional logic with propositional quantifiers, denoted by $\mathrm{SCl}_{Q}$. We prove that $\mathrm{SCl}_{\mathrm{Q}}$ does not have the finite model property and that it is undecidable. We also present examples of how to interpret in $\mathrm{SCl}_{\mathrm{Q}}$ various mathematical theories, such as the theory of groups, rings, and fields, and we characterize the spectra of $\mathrm{SCl}_{\mathrm{Q}}$-sentences. Finally, we present a translation of $\mathrm{SCl}_{\mathrm{Q}}$ into a classical two-sorted first-order logic, and we use the translation to prove some model-theoretic properties of $\mathrm{SCl}_{\mathrm{Q}}$.
\end{abstract}

\section{Introduction}

Non-Fregean logics, introduced by Roman Suszko, can be seen as a realization of Gottlob Frege's semantic program with the exception of a postulate-known in the literature as the Fregean axiom - that treats the truth value of a sentence as its denotation. According to Frege, sentences are not only true or false, but are also names denoting the corresponding truth values. The Fregean axiom is a fundamental assumption underlying classical logic. The theory of models based on classical logic does not involve a universe corresponding to the statements in the appropriate language, but only assigns a simple truth value to each statement. Thus, the Fregean axiom is a formal expression of a certain philosophical view concerning the meanings of statements.

Non-Fregean logic was explicitly proposed by Suszko as an alternative to the established standard. Non-Fregean logic rejects the Fregean axiom and introduces a universe of the semantic correlates of statements, known as the universe of situations. In order to express claims concerning the universe of situations, a new connective $\equiv$, called the identity connective, is added to the language. The identity connective, as the name implies, expresses the identity of two statements; that is, it

Received January 21, 2013; accepted November 7, 2013

First published online February 9, 2016

2010 Mathematics Subject Classification: Primary 03B60; Secondary 03C80, 68Q19

Keywords: non-Fregean logic, sentential calculus with identity, identity connective, situational semantics

(C) 2016 by University of Notre Dame 10.1215/00294527-3470547 


\title{
Non-Fregean Propositional Logic with Quantifiers
}

\author{
Joanna Golińska-Pilarek and Taneli Huuskonen
}

\begin{abstract}
We study the non-Fregean propositional logic with propositional quantifiers, denoted by $\mathrm{SCl}_{Q}$. We prove that $\mathrm{SCl}_{\mathrm{Q}}$ does not have the finite model property and that it is undecidable. We also present examples of how to interpret in $\mathrm{SCl}_{\mathrm{Q}}$ various mathematical theories, such as the theory of groups, rings, and fields, and we characterize the spectra of $\mathrm{SCl}_{\mathrm{Q}}$-sentences. Finally, we present a translation of $\mathrm{SCl}_{\mathrm{Q}}$ into a classical two-sorted first-order logic, and we use the translation to prove some model-theoretic properties of $\mathrm{SCl}_{\mathrm{Q}}$.
\end{abstract}

\section{Introduction}

Non-Fregean logics, introduced by Roman Suszko, can be seen as a realization of Gottlob Frege's semantic program with the exception of a postulate-known in the literature as the Fregean axiom - that treats the truth value of a sentence as its denotation. According to Frege, sentences are not only true or false, but are also names denoting the corresponding truth values. The Fregean axiom is a fundamental assumption underlying classical logic. The theory of models based on classical logic does not involve a universe corresponding to the statements in the appropriate language, but only assigns a simple truth value to each statement. Thus, the Fregean axiom is a formal expression of a certain philosophical view concerning the meanings of statements.

Non-Fregean logic was explicitly proposed by Suszko as an alternative to the established standard. Non-Fregean logic rejects the Fregean axiom and introduces a universe of the semantic correlates of statements, known as the universe of situations. In order to express claims concerning the universe of situations, a new connective $\equiv$, called the identity connective, is added to the language. The identity connective, as the name implies, expresses the identity of two statements; that is, it

Received January 21, 2013; accepted November 7, 2013

First published online February 9, 2016

2010 Mathematics Subject Classification: Primary 03B60; Secondary 03C80, 68Q19

Keywords: non-Fregean logic, sentential calculus with identity, identity connective, situational semantics

(C) 2016 by University of Notre Dame 10.1215/00294527-3470547 


\title{
Non-Fregean Propositional Logic with Quantifiers
}

\author{
Joanna Golińska-Pilarek and Taneli Huuskonen
}

\begin{abstract}
We study the non-Fregean propositional logic with propositional quantifiers, denoted by $\mathrm{SCl}_{Q}$. We prove that $\mathrm{SCl}_{\mathrm{Q}}$ does not have the finite model property and that it is undecidable. We also present examples of how to interpret in $\mathrm{SCl}_{\mathrm{Q}}$ various mathematical theories, such as the theory of groups, rings, and fields, and we characterize the spectra of $\mathrm{SCl}_{\mathrm{Q}}$-sentences. Finally, we present a translation of $\mathrm{SCl}_{\mathrm{Q}}$ into a classical two-sorted first-order logic, and we use the translation to prove some model-theoretic properties of $\mathrm{SCl}_{\mathrm{Q}}$.
\end{abstract}

\section{Introduction}

Non-Fregean logics, introduced by Roman Suszko, can be seen as a realization of Gottlob Frege's semantic program with the exception of a postulate-known in the literature as the Fregean axiom - that treats the truth value of a sentence as its denotation. According to Frege, sentences are not only true or false, but are also names denoting the corresponding truth values. The Fregean axiom is a fundamental assumption underlying classical logic. The theory of models based on classical logic does not involve a universe corresponding to the statements in the appropriate language, but only assigns a simple truth value to each statement. Thus, the Fregean axiom is a formal expression of a certain philosophical view concerning the meanings of statements.

Non-Fregean logic was explicitly proposed by Suszko as an alternative to the established standard. Non-Fregean logic rejects the Fregean axiom and introduces a universe of the semantic correlates of statements, known as the universe of situations. In order to express claims concerning the universe of situations, a new connective $\equiv$, called the identity connective, is added to the language. The identity connective, as the name implies, expresses the identity of two statements; that is, it

Received January 21, 2013; accepted November 7, 2013

First published online February 9, 2016

2010 Mathematics Subject Classification: Primary 03B60; Secondary 03C80, 68Q19

Keywords: non-Fregean logic, sentential calculus with identity, identity connective, situational semantics

(C) 2016 by University of Notre Dame 10.1215/00294527-3470547 


\title{
Non-Fregean Propositional Logic with Quantifiers
}

\author{
Joanna Golińska-Pilarek and Taneli Huuskonen
}

\begin{abstract}
We study the non-Fregean propositional logic with propositional quantifiers, denoted by $\mathrm{SCl}_{Q}$. We prove that $\mathrm{SCl}_{\mathrm{Q}}$ does not have the finite model property and that it is undecidable. We also present examples of how to interpret in $\mathrm{SCl}_{\mathrm{Q}}$ various mathematical theories, such as the theory of groups, rings, and fields, and we characterize the spectra of $\mathrm{SCl}_{\mathrm{Q}}$-sentences. Finally, we present a translation of $\mathrm{SCl}_{\mathrm{Q}}$ into a classical two-sorted first-order logic, and we use the translation to prove some model-theoretic properties of $\mathrm{SCl}_{\mathrm{Q}}$.
\end{abstract}

\section{Introduction}

Non-Fregean logics, introduced by Roman Suszko, can be seen as a realization of Gottlob Frege's semantic program with the exception of a postulate-known in the literature as the Fregean axiom - that treats the truth value of a sentence as its denotation. According to Frege, sentences are not only true or false, but are also names denoting the corresponding truth values. The Fregean axiom is a fundamental assumption underlying classical logic. The theory of models based on classical logic does not involve a universe corresponding to the statements in the appropriate language, but only assigns a simple truth value to each statement. Thus, the Fregean axiom is a formal expression of a certain philosophical view concerning the meanings of statements.

Non-Fregean logic was explicitly proposed by Suszko as an alternative to the established standard. Non-Fregean logic rejects the Fregean axiom and introduces a universe of the semantic correlates of statements, known as the universe of situations. In order to express claims concerning the universe of situations, a new connective $\equiv$, called the identity connective, is added to the language. The identity connective, as the name implies, expresses the identity of two statements; that is, it

Received January 21, 2013; accepted November 7, 2013

First published online February 9, 2016

2010 Mathematics Subject Classification: Primary 03B60; Secondary 03C80, 68Q19

Keywords: non-Fregean logic, sentential calculus with identity, identity connective, situational semantics

(C) 2016 by University of Notre Dame 10.1215/00294527-3470547 


\title{
Non-Fregean Propositional Logic with Quantifiers
}

\author{
Joanna Golińska-Pilarek and Taneli Huuskonen
}

\begin{abstract}
We study the non-Fregean propositional logic with propositional quantifiers, denoted by $\mathrm{SCl}_{Q}$. We prove that $\mathrm{SCl}_{\mathrm{Q}}$ does not have the finite model property and that it is undecidable. We also present examples of how to interpret in $\mathrm{SCl}_{\mathrm{Q}}$ various mathematical theories, such as the theory of groups, rings, and fields, and we characterize the spectra of $\mathrm{SCl}_{\mathrm{Q}}$-sentences. Finally, we present a translation of $\mathrm{SCl}_{\mathrm{Q}}$ into a classical two-sorted first-order logic, and we use the translation to prove some model-theoretic properties of $\mathrm{SCl}_{\mathrm{Q}}$.
\end{abstract}

\section{Introduction}

Non-Fregean logics, introduced by Roman Suszko, can be seen as a realization of Gottlob Frege's semantic program with the exception of a postulate-known in the literature as the Fregean axiom - that treats the truth value of a sentence as its denotation. According to Frege, sentences are not only true or false, but are also names denoting the corresponding truth values. The Fregean axiom is a fundamental assumption underlying classical logic. The theory of models based on classical logic does not involve a universe corresponding to the statements in the appropriate language, but only assigns a simple truth value to each statement. Thus, the Fregean axiom is a formal expression of a certain philosophical view concerning the meanings of statements.

Non-Fregean logic was explicitly proposed by Suszko as an alternative to the established standard. Non-Fregean logic rejects the Fregean axiom and introduces a universe of the semantic correlates of statements, known as the universe of situations. In order to express claims concerning the universe of situations, a new connective $\equiv$, called the identity connective, is added to the language. The identity connective, as the name implies, expresses the identity of two statements; that is, it

Received January 21, 2013; accepted November 7, 2013

First published online February 9, 2016

2010 Mathematics Subject Classification: Primary 03B60; Secondary 03C80, 68Q19

Keywords: non-Fregean logic, sentential calculus with identity, identity connective, situational semantics

(C) 2016 by University of Notre Dame 10.1215/00294527-3470547 


\title{
Non-Fregean Propositional Logic with Quantifiers
}

\author{
Joanna Golińska-Pilarek and Taneli Huuskonen
}

\begin{abstract}
We study the non-Fregean propositional logic with propositional quantifiers, denoted by $\mathrm{SCl}_{Q}$. We prove that $\mathrm{SCl}_{\mathrm{Q}}$ does not have the finite model property and that it is undecidable. We also present examples of how to interpret in $\mathrm{SCl}_{\mathrm{Q}}$ various mathematical theories, such as the theory of groups, rings, and fields, and we characterize the spectra of $\mathrm{SCl}_{\mathrm{Q}}$-sentences. Finally, we present a translation of $\mathrm{SCl}_{\mathrm{Q}}$ into a classical two-sorted first-order logic, and we use the translation to prove some model-theoretic properties of $\mathrm{SCl}_{\mathrm{Q}}$.
\end{abstract}

\section{Introduction}

Non-Fregean logics, introduced by Roman Suszko, can be seen as a realization of Gottlob Frege's semantic program with the exception of a postulate-known in the literature as the Fregean axiom - that treats the truth value of a sentence as its denotation. According to Frege, sentences are not only true or false, but are also names denoting the corresponding truth values. The Fregean axiom is a fundamental assumption underlying classical logic. The theory of models based on classical logic does not involve a universe corresponding to the statements in the appropriate language, but only assigns a simple truth value to each statement. Thus, the Fregean axiom is a formal expression of a certain philosophical view concerning the meanings of statements.

Non-Fregean logic was explicitly proposed by Suszko as an alternative to the established standard. Non-Fregean logic rejects the Fregean axiom and introduces a universe of the semantic correlates of statements, known as the universe of situations. In order to express claims concerning the universe of situations, a new connective $\equiv$, called the identity connective, is added to the language. The identity connective, as the name implies, expresses the identity of two statements; that is, it

Received January 21, 2013; accepted November 7, 2013

First published online February 9, 2016

2010 Mathematics Subject Classification: Primary 03B60; Secondary 03C80, 68Q19

Keywords: non-Fregean logic, sentential calculus with identity, identity connective, situational semantics

(C) 2016 by University of Notre Dame 10.1215/00294527-3470547 


\title{
Non-Fregean Propositional Logic with Quantifiers
}

\author{
Joanna Golińska-Pilarek and Taneli Huuskonen
}

\begin{abstract}
We study the non-Fregean propositional logic with propositional quantifiers, denoted by $\mathrm{SCl}_{Q}$. We prove that $\mathrm{SCl}_{\mathrm{Q}}$ does not have the finite model property and that it is undecidable. We also present examples of how to interpret in $\mathrm{SCl}_{\mathrm{Q}}$ various mathematical theories, such as the theory of groups, rings, and fields, and we characterize the spectra of $\mathrm{SCl}_{\mathrm{Q}}$-sentences. Finally, we present a translation of $\mathrm{SCl}_{\mathrm{Q}}$ into a classical two-sorted first-order logic, and we use the translation to prove some model-theoretic properties of $\mathrm{SCl}_{\mathrm{Q}}$.
\end{abstract}

\section{Introduction}

Non-Fregean logics, introduced by Roman Suszko, can be seen as a realization of Gottlob Frege's semantic program with the exception of a postulate-known in the literature as the Fregean axiom - that treats the truth value of a sentence as its denotation. According to Frege, sentences are not only true or false, but are also names denoting the corresponding truth values. The Fregean axiom is a fundamental assumption underlying classical logic. The theory of models based on classical logic does not involve a universe corresponding to the statements in the appropriate language, but only assigns a simple truth value to each statement. Thus, the Fregean axiom is a formal expression of a certain philosophical view concerning the meanings of statements.

Non-Fregean logic was explicitly proposed by Suszko as an alternative to the established standard. Non-Fregean logic rejects the Fregean axiom and introduces a universe of the semantic correlates of statements, known as the universe of situations. In order to express claims concerning the universe of situations, a new connective $\equiv$, called the identity connective, is added to the language. The identity connective, as the name implies, expresses the identity of two statements; that is, it

Received January 21, 2013; accepted November 7, 2013

First published online February 9, 2016

2010 Mathematics Subject Classification: Primary 03B60; Secondary 03C80, 68Q19

Keywords: non-Fregean logic, sentential calculus with identity, identity connective, situational semantics

(C) 2016 by University of Notre Dame 10.1215/00294527-3470547 


\title{
Non-Fregean Propositional Logic with Quantifiers
}

\author{
Joanna Golińska-Pilarek and Taneli Huuskonen
}

\begin{abstract}
We study the non-Fregean propositional logic with propositional quantifiers, denoted by $\mathrm{SCl}_{Q}$. We prove that $\mathrm{SCl}_{\mathrm{Q}}$ does not have the finite model property and that it is undecidable. We also present examples of how to interpret in $\mathrm{SCl}_{\mathrm{Q}}$ various mathematical theories, such as the theory of groups, rings, and fields, and we characterize the spectra of $\mathrm{SCl}_{\mathrm{Q}}$-sentences. Finally, we present a translation of $\mathrm{SCl}_{\mathrm{Q}}$ into a classical two-sorted first-order logic, and we use the translation to prove some model-theoretic properties of $\mathrm{SCl}_{\mathrm{Q}}$.
\end{abstract}

\section{Introduction}

Non-Fregean logics, introduced by Roman Suszko, can be seen as a realization of Gottlob Frege's semantic program with the exception of a postulate-known in the literature as the Fregean axiom - that treats the truth value of a sentence as its denotation. According to Frege, sentences are not only true or false, but are also names denoting the corresponding truth values. The Fregean axiom is a fundamental assumption underlying classical logic. The theory of models based on classical logic does not involve a universe corresponding to the statements in the appropriate language, but only assigns a simple truth value to each statement. Thus, the Fregean axiom is a formal expression of a certain philosophical view concerning the meanings of statements.

Non-Fregean logic was explicitly proposed by Suszko as an alternative to the established standard. Non-Fregean logic rejects the Fregean axiom and introduces a universe of the semantic correlates of statements, known as the universe of situations. In order to express claims concerning the universe of situations, a new connective $\equiv$, called the identity connective, is added to the language. The identity connective, as the name implies, expresses the identity of two statements; that is, it

Received January 21, 2013; accepted November 7, 2013

First published online February 9, 2016

2010 Mathematics Subject Classification: Primary 03B60; Secondary 03C80, 68Q19

Keywords: non-Fregean logic, sentential calculus with identity, identity connective, situational semantics

(C) 2016 by University of Notre Dame 10.1215/00294527-3470547 


\title{
Non-Fregean Propositional Logic with Quantifiers
}

\author{
Joanna Golińska-Pilarek and Taneli Huuskonen
}

\begin{abstract}
We study the non-Fregean propositional logic with propositional quantifiers, denoted by $\mathrm{SCl}_{Q}$. We prove that $\mathrm{SCl}_{\mathrm{Q}}$ does not have the finite model property and that it is undecidable. We also present examples of how to interpret in $\mathrm{SCl}_{\mathrm{Q}}$ various mathematical theories, such as the theory of groups, rings, and fields, and we characterize the spectra of $\mathrm{SCl}_{\mathrm{Q}}$-sentences. Finally, we present a translation of $\mathrm{SCl}_{\mathrm{Q}}$ into a classical two-sorted first-order logic, and we use the translation to prove some model-theoretic properties of $\mathrm{SCl}_{\mathrm{Q}}$.
\end{abstract}

\section{Introduction}

Non-Fregean logics, introduced by Roman Suszko, can be seen as a realization of Gottlob Frege's semantic program with the exception of a postulate-known in the literature as the Fregean axiom - that treats the truth value of a sentence as its denotation. According to Frege, sentences are not only true or false, but are also names denoting the corresponding truth values. The Fregean axiom is a fundamental assumption underlying classical logic. The theory of models based on classical logic does not involve a universe corresponding to the statements in the appropriate language, but only assigns a simple truth value to each statement. Thus, the Fregean axiom is a formal expression of a certain philosophical view concerning the meanings of statements.

Non-Fregean logic was explicitly proposed by Suszko as an alternative to the established standard. Non-Fregean logic rejects the Fregean axiom and introduces a universe of the semantic correlates of statements, known as the universe of situations. In order to express claims concerning the universe of situations, a new connective $\equiv$, called the identity connective, is added to the language. The identity connective, as the name implies, expresses the identity of two statements; that is, it

Received January 21, 2013; accepted November 7, 2013

First published online February 9, 2016

2010 Mathematics Subject Classification: Primary 03B60; Secondary 03C80, 68Q19

Keywords: non-Fregean logic, sentential calculus with identity, identity connective, situational semantics

(C) 2016 by University of Notre Dame 10.1215/00294527-3470547 


\title{
Non-Fregean Propositional Logic with Quantifiers
}

\author{
Joanna Golińska-Pilarek and Taneli Huuskonen
}

\begin{abstract}
We study the non-Fregean propositional logic with propositional quantifiers, denoted by $\mathrm{SCl}_{Q}$. We prove that $\mathrm{SCl}_{\mathrm{Q}}$ does not have the finite model property and that it is undecidable. We also present examples of how to interpret in $\mathrm{SCl}_{\mathrm{Q}}$ various mathematical theories, such as the theory of groups, rings, and fields, and we characterize the spectra of $\mathrm{SCl}_{\mathrm{Q}}$-sentences. Finally, we present a translation of $\mathrm{SCl}_{\mathrm{Q}}$ into a classical two-sorted first-order logic, and we use the translation to prove some model-theoretic properties of $\mathrm{SCl}_{\mathrm{Q}}$.
\end{abstract}

\section{Introduction}

Non-Fregean logics, introduced by Roman Suszko, can be seen as a realization of Gottlob Frege's semantic program with the exception of a postulate-known in the literature as the Fregean axiom - that treats the truth value of a sentence as its denotation. According to Frege, sentences are not only true or false, but are also names denoting the corresponding truth values. The Fregean axiom is a fundamental assumption underlying classical logic. The theory of models based on classical logic does not involve a universe corresponding to the statements in the appropriate language, but only assigns a simple truth value to each statement. Thus, the Fregean axiom is a formal expression of a certain philosophical view concerning the meanings of statements.

Non-Fregean logic was explicitly proposed by Suszko as an alternative to the established standard. Non-Fregean logic rejects the Fregean axiom and introduces a universe of the semantic correlates of statements, known as the universe of situations. In order to express claims concerning the universe of situations, a new connective $\equiv$, called the identity connective, is added to the language. The identity connective, as the name implies, expresses the identity of two statements; that is, it

Received January 21, 2013; accepted November 7, 2013

First published online February 9, 2016

2010 Mathematics Subject Classification: Primary 03B60; Secondary 03C80, 68Q19

Keywords: non-Fregean logic, sentential calculus with identity, identity connective, situational semantics

(C) 2016 by University of Notre Dame 10.1215/00294527-3470547 


\title{
Non-Fregean Propositional Logic with Quantifiers
}

\author{
Joanna Golińska-Pilarek and Taneli Huuskonen
}

\begin{abstract}
We study the non-Fregean propositional logic with propositional quantifiers, denoted by $\mathrm{SCl}_{Q}$. We prove that $\mathrm{SCl}_{\mathrm{Q}}$ does not have the finite model property and that it is undecidable. We also present examples of how to interpret in $\mathrm{SCl}_{\mathrm{Q}}$ various mathematical theories, such as the theory of groups, rings, and fields, and we characterize the spectra of $\mathrm{SCl}_{\mathrm{Q}}$-sentences. Finally, we present a translation of $\mathrm{SCl}_{\mathrm{Q}}$ into a classical two-sorted first-order logic, and we use the translation to prove some model-theoretic properties of $\mathrm{SCl}_{\mathrm{Q}}$.
\end{abstract}

\section{Introduction}

Non-Fregean logics, introduced by Roman Suszko, can be seen as a realization of Gottlob Frege's semantic program with the exception of a postulate-known in the literature as the Fregean axiom - that treats the truth value of a sentence as its denotation. According to Frege, sentences are not only true or false, but are also names denoting the corresponding truth values. The Fregean axiom is a fundamental assumption underlying classical logic. The theory of models based on classical logic does not involve a universe corresponding to the statements in the appropriate language, but only assigns a simple truth value to each statement. Thus, the Fregean axiom is a formal expression of a certain philosophical view concerning the meanings of statements.

Non-Fregean logic was explicitly proposed by Suszko as an alternative to the established standard. Non-Fregean logic rejects the Fregean axiom and introduces a universe of the semantic correlates of statements, known as the universe of situations. In order to express claims concerning the universe of situations, a new connective $\equiv$, called the identity connective, is added to the language. The identity connective, as the name implies, expresses the identity of two statements; that is, it

Received January 21, 2013; accepted November 7, 2013

First published online February 9, 2016

2010 Mathematics Subject Classification: Primary 03B60; Secondary 03C80, 68Q19

Keywords: non-Fregean logic, sentential calculus with identity, identity connective, situational semantics

(C) 2016 by University of Notre Dame 10.1215/00294527-3470547 


\title{
Non-Fregean Propositional Logic with Quantifiers
}

\author{
Joanna Golińska-Pilarek and Taneli Huuskonen
}

\begin{abstract}
We study the non-Fregean propositional logic with propositional quantifiers, denoted by $\mathrm{SCl}_{Q}$. We prove that $\mathrm{SCl}_{\mathrm{Q}}$ does not have the finite model property and that it is undecidable. We also present examples of how to interpret in $\mathrm{SCl}_{\mathrm{Q}}$ various mathematical theories, such as the theory of groups, rings, and fields, and we characterize the spectra of $\mathrm{SCl}_{\mathrm{Q}}$-sentences. Finally, we present a translation of $\mathrm{SCl}_{\mathrm{Q}}$ into a classical two-sorted first-order logic, and we use the translation to prove some model-theoretic properties of $\mathrm{SCl}_{\mathrm{Q}}$.
\end{abstract}

\section{Introduction}

Non-Fregean logics, introduced by Roman Suszko, can be seen as a realization of Gottlob Frege's semantic program with the exception of a postulate-known in the literature as the Fregean axiom - that treats the truth value of a sentence as its denotation. According to Frege, sentences are not only true or false, but are also names denoting the corresponding truth values. The Fregean axiom is a fundamental assumption underlying classical logic. The theory of models based on classical logic does not involve a universe corresponding to the statements in the appropriate language, but only assigns a simple truth value to each statement. Thus, the Fregean axiom is a formal expression of a certain philosophical view concerning the meanings of statements.

Non-Fregean logic was explicitly proposed by Suszko as an alternative to the established standard. Non-Fregean logic rejects the Fregean axiom and introduces a universe of the semantic correlates of statements, known as the universe of situations. In order to express claims concerning the universe of situations, a new connective $\equiv$, called the identity connective, is added to the language. The identity connective, as the name implies, expresses the identity of two statements; that is, it

Received January 21, 2013; accepted November 7, 2013

First published online February 9, 2016

2010 Mathematics Subject Classification: Primary 03B60; Secondary 03C80, 68Q19

Keywords: non-Fregean logic, sentential calculus with identity, identity connective, situational semantics

(C) 2016 by University of Notre Dame 10.1215/00294527-3470547 


\title{
Non-Fregean Propositional Logic with Quantifiers
}

\author{
Joanna Golińska-Pilarek and Taneli Huuskonen
}

\begin{abstract}
We study the non-Fregean propositional logic with propositional quantifiers, denoted by $\mathrm{SCl}_{Q}$. We prove that $\mathrm{SCl}_{\mathrm{Q}}$ does not have the finite model property and that it is undecidable. We also present examples of how to interpret in $\mathrm{SCl}_{\mathrm{Q}}$ various mathematical theories, such as the theory of groups, rings, and fields, and we characterize the spectra of $\mathrm{SCl}_{\mathrm{Q}}$-sentences. Finally, we present a translation of $\mathrm{SCl}_{\mathrm{Q}}$ into a classical two-sorted first-order logic, and we use the translation to prove some model-theoretic properties of $\mathrm{SCl}_{\mathrm{Q}}$.
\end{abstract}

\section{Introduction}

Non-Fregean logics, introduced by Roman Suszko, can be seen as a realization of Gottlob Frege's semantic program with the exception of a postulate-known in the literature as the Fregean axiom - that treats the truth value of a sentence as its denotation. According to Frege, sentences are not only true or false, but are also names denoting the corresponding truth values. The Fregean axiom is a fundamental assumption underlying classical logic. The theory of models based on classical logic does not involve a universe corresponding to the statements in the appropriate language, but only assigns a simple truth value to each statement. Thus, the Fregean axiom is a formal expression of a certain philosophical view concerning the meanings of statements.

Non-Fregean logic was explicitly proposed by Suszko as an alternative to the established standard. Non-Fregean logic rejects the Fregean axiom and introduces a universe of the semantic correlates of statements, known as the universe of situations. In order to express claims concerning the universe of situations, a new connective $\equiv$, called the identity connective, is added to the language. The identity connective, as the name implies, expresses the identity of two statements; that is, it

Received January 21, 2013; accepted November 7, 2013

First published online February 9, 2016

2010 Mathematics Subject Classification: Primary 03B60; Secondary 03C80, 68Q19

Keywords: non-Fregean logic, sentential calculus with identity, identity connective, situational semantics

(C) 2016 by University of Notre Dame 10.1215/00294527-3470547 


\title{
Non-Fregean Propositional Logic with Quantifiers
}

\author{
Joanna Golińska-Pilarek and Taneli Huuskonen
}

\begin{abstract}
We study the non-Fregean propositional logic with propositional quantifiers, denoted by $\mathrm{SCl}_{Q}$. We prove that $\mathrm{SCl}_{\mathrm{Q}}$ does not have the finite model property and that it is undecidable. We also present examples of how to interpret in $\mathrm{SCl}_{\mathrm{Q}}$ various mathematical theories, such as the theory of groups, rings, and fields, and we characterize the spectra of $\mathrm{SCl}_{\mathrm{Q}}$-sentences. Finally, we present a translation of $\mathrm{SCl}_{\mathrm{Q}}$ into a classical two-sorted first-order logic, and we use the translation to prove some model-theoretic properties of $\mathrm{SCl}_{\mathrm{Q}}$.
\end{abstract}

\section{Introduction}

Non-Fregean logics, introduced by Roman Suszko, can be seen as a realization of Gottlob Frege's semantic program with the exception of a postulate-known in the literature as the Fregean axiom - that treats the truth value of a sentence as its denotation. According to Frege, sentences are not only true or false, but are also names denoting the corresponding truth values. The Fregean axiom is a fundamental assumption underlying classical logic. The theory of models based on classical logic does not involve a universe corresponding to the statements in the appropriate language, but only assigns a simple truth value to each statement. Thus, the Fregean axiom is a formal expression of a certain philosophical view concerning the meanings of statements.

Non-Fregean logic was explicitly proposed by Suszko as an alternative to the established standard. Non-Fregean logic rejects the Fregean axiom and introduces a universe of the semantic correlates of statements, known as the universe of situations. In order to express claims concerning the universe of situations, a new connective $\equiv$, called the identity connective, is added to the language. The identity connective, as the name implies, expresses the identity of two statements; that is, it

Received January 21, 2013; accepted November 7, 2013

First published online February 9, 2016

2010 Mathematics Subject Classification: Primary 03B60; Secondary 03C80, 68Q19

Keywords: non-Fregean logic, sentential calculus with identity, identity connective, situational semantics

(C) 2016 by University of Notre Dame 10.1215/00294527-3470547 